\title{
Flows along penumbral flux tubes in sunspots
}

\section{Instability of super-Alfvénic, serpentine solutions}

\begin{abstract}
J. H. Thomas
Department of Mechanical Engineering and Department of Physics and Astronomy, University of Rochester, Rochester, NY 14627-0171, USA

e-mail: thomas@me.rochester.edu

Received 9 May 2005 / Accepted 9 July 2005

Abstract. The super-Alfvénic, undulating "sea-serpent" flow configurations along thin magnetic flux tubes, proposed by Schlichenmaier (2002, Astron. Nachr., 323, 303; 2003, ASP Conf. Ser., 286, 24) to explain both the Evershed flow and moving penumbral grains in a sunspot, are shown to be gravitationally unstable. Any undulations that occur for super-Alfvénic flow speeds will form preferentially in a horizontal plane and hence will not explain Evershed downflows or outward-moving penumbral grains. Sub-Alfvénic, arched flow configurations, on the other hand, are gravitationally stable.
\end{abstract}

Key words. sunspots - Sun: magnetic fields - magnetohydrodynamics (MHD)

\section{Introduction}

In recent years significant advances in high-resolution observations have stimulated theoretical modeling of the structure and dynamics of the enigmatic sunspot penumbra, with its complex arrangement of magnetic fields and fluid motions (Solanki 2003; Thomas \& Weiss 2004). A long-standing problem in this regard has been to explain the Evershed flow, a radial, nearly horizontal outflow of gas in the penumbra. Although there have been other interpretations, the general theoretical model that best fits the observations consists of flows along individual magnetic flux tubes driven by a pressure difference. Models in this category are based on the thin flux tube approximation and include steady flows (often called "siphon" flows: Meyer \& Schmidt 1968; Thomas 1988; Degenhardt 1989, 1991; Thomas \& Montesinos 1990, 1991, 1993; Montesinos \& Thomas 1997) and time-dependent flows (Schlichenmaier et al. 1998a,b; Schlichenmaier 2002, 2003).

Here we examine the gravitational stability of the "moving tube" model of Schlichenmaier et al. (1998a,b), hereafter to be called the SJS model, and the steady siphon-flow model as exemplified by the models Thomas \& Montesinos (1993) and Montesinos \& Thomas (1997), hereafter to be called the TM model. We show that the serpentine tube configurations with fast, super-Alfvénic flows, based on the SJS model and proposed by Schlichenmaier $(2002,2003)$ to explain both the Evershed flow and the behavior of the bright penumbral grains, are gravitationally unstable to displacements out of the vertical plane and hence will not occur. On the other hand, the subAlfvénic, arched siphon-flow flux tubes in the TM model are shown to be stable to such displacements.
Figure 1 shows schematic representations of the flux tube path for a typical "normal" configuration (non-undulating) of the SJS model and a typical arched configuration of the TM siphon-flow model. In the thin flux tube approximation (common to the SJS and TM models), it is assumed that the flux tube is everywhere in lateral pressure balance, with the same total pressure (gas plus magnetic) inside and outside the tube, i.e.,

$p+\frac{B^{2}}{8 \pi}=p_{\mathrm{e}}+\frac{B_{\mathrm{e}}^{2}}{8 \pi}$

where the subscript "e" denotes quantities in the external atmosphere. In the TM model, the arched flux tube has both an upstream and a downstream footpoint at the solar surface, and the flow is driven because of a reduced internal gas pressure (higher internal magnetic pressure) at the downstream footpoint, which corresponds to an intense magnetic flux concentration near the outer boundary of the penumbra. In this case, we have $p<p_{\mathrm{e}}$ all along the tube, so $B>B_{\mathrm{e}}$ all along the tube. In the SJS model, there is no downstream footpoint: the tube continues outward along a magnetic canopy outside the sunspot to some point where an open boundary condition is applied. (Since observations show that only a small fraction of the Evershed flow continues outward along the canopy, the SJS model has difficulties in this regard.) In the SJS model, the flow is driven because of an increased pressure at the upstream footpoint, and hence $p>p_{\mathrm{e}}$ and $B<B_{\mathrm{e}}$ all along the tube.

Observations have revealed that much of the Evershed flow occurs along "returning" flux tubes that dive back down below the solar surface near the outer edge of the penumbra (see Thomas \& Weiss 2004, Sect. 5.4.1, and references therein). 

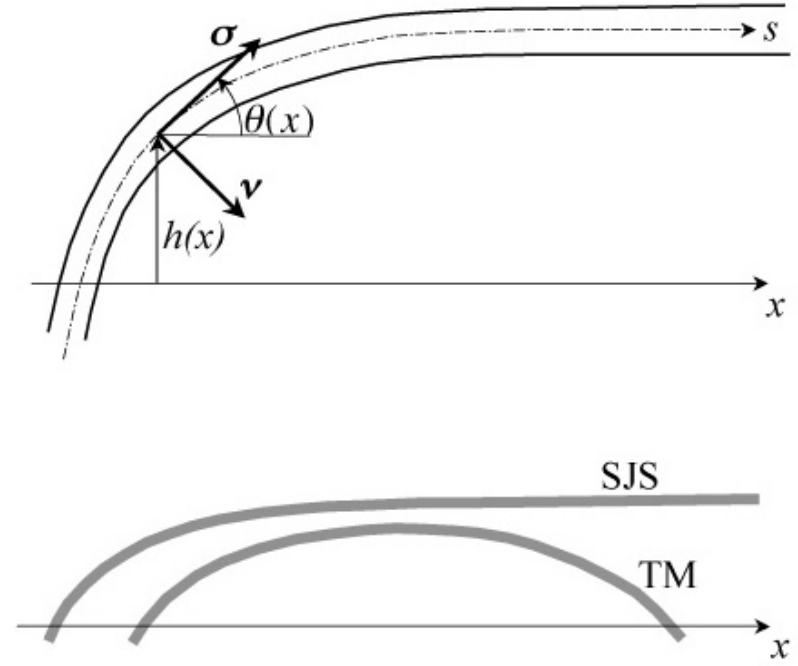

Fig. 1. (Above) coordinate system for a thin penumbral flux tube. (Below) schematic representations of a typical "normal" flux tube configuration (non-serpentine) of the SJS model and a typical arched flux tube configuration of the TM siphon-flow model.

The "normal" configuration of the SJS model, as shown in Fig. 1, cannot explain such downflows. Therefore, Schlichenmaier $(2002,2003)$ has proposed that another form of solution found in the SJS model, in which the flux tube assumes a vertically undulating, serpentine form, is the preferred mode. Figure 2 shows a schematic representation of this configuration (compare, for example, with Figs. 2-5 of Schlichenmaier 2002 or Fig. 3 of Schlichenmaier 2003). An inherent feature of these serpentine solutions is that the flow speed is everywhere greater than the Alfvén speed within the tube, i.e., the flow is superAlfvénic, and hence also supercritical ${ }^{1}$, everywhere along the undulating tube. The flow decelerates in the rising portions of the tube and accelerates in the descending portions, while remaining everywhere super-Alfvénic. In the TM model, on the other hand, because the tube re-enters the Sun at the downstream footpoint where the gas pressure is again high, the flow is decelerated there, or in the case of a supercritical flow, is slowed abruptly to subcritical speed at a tube shock somewhere along the downstream leg of the arch (Thomas \& Montesinos 1991).

\section{Basic equations}

Here we give a brief derivation of the basic equations describing a steady flow along a thin flux tube and the equilibrium path of the flux tube through the surrounding atmosphere (see also Spruit 1981; Thomas \& Montesinos 1990). We assume that the flux tube is thin, in the sense that its radius is much smaller than either the scale height of the surrounding atmosphere or the radius of curvature of the axis of the flux tube. Then the flow inside the tube can be considered one-dimensional, i.e., locally

${ }^{1}$ A pressure pulse travels along a thin magnetic flux tube at the tube speed $c_{\mathrm{t}}=\sqrt{c^{2} a^{2} /\left(c^{2}+a^{2}\right)}$, where $c$ is the internal sound speed and $a$ is the internal Alfvén speed. Hence, the tube speed $c_{\mathrm{t}}$ replaces the sound speed $c$ as the critical speed for flow along the tube. Note also that $c_{\mathrm{t}}<\min (c, a)$.
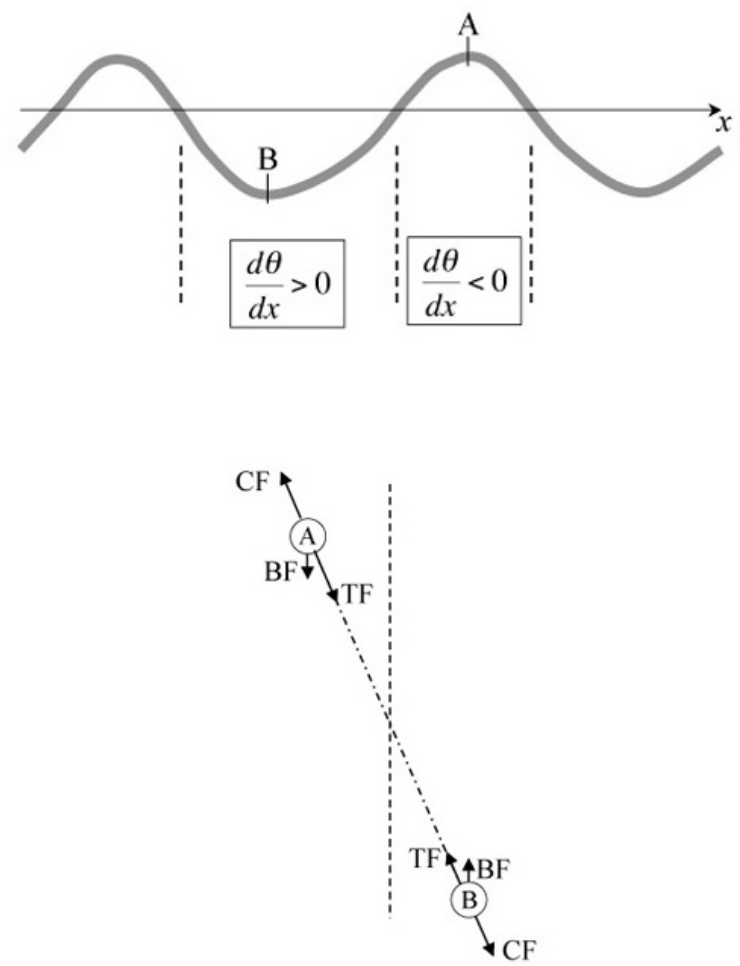

Fig. 2. (Above) schematic diagram of a serpentine flux tube configuration of Schlichenmaier $(2002,2003)$, with sections of downward and upward concavity marked. (Below) sketch showing the plane of the axis of the serpentine configuration tilted away from the vertical. Also shown is the force balance among the centrifugal force (CF), the magnetic tension force (TF), and the buoyancy force (BF) at a high point (A) and low point (B) along the serpentine configuration.

parallel to the tube axis and uniform across the cross-section of the tube, so that all flow variables are functions only of the tangential coordinate $s$. Referring to Fig. 1, we assume that the flux tube lies in a vertical plane, and we let $\sigma$ denote the unit vector in the tangential $(s)$ direction, pointing in the direction of the flow, and $v$ denote the unit vector normal to the axis of the flux tube, pointing $90^{\circ}$ clockwise from $\sigma$.

The siphon flows computed by TM are steady-state solutions. The flows in the SJS model are time dependent, but the evolution of the serpentine configuration is slow compared to the passage of individual fluid particles, and hence the flow can be considered as quasi-steady (as has indeed been done by Schlichenmaier (2002, 2003, himself). Thus, the balance of forces can be discussed in terms of a steady flow.

The momentum equation for inviscid steady flow is

$$
\begin{aligned}
\rho \boldsymbol{v} \cdot \nabla \boldsymbol{v}=-\nabla p & +\rho \boldsymbol{g}+\frac{1}{4 \pi}(\nabla \times \boldsymbol{B}) \times \boldsymbol{B}= \\
& -\nabla\left(p+\frac{B^{2}}{8 \pi}\right)+\rho \boldsymbol{g}+\frac{1}{4 \pi} \boldsymbol{B} \cdot \nabla \boldsymbol{B} .
\end{aligned}
$$

As in the SJS model, we shall assume that the magnetic field $\boldsymbol{B}_{\mathrm{e}}$ in the external atmosphere is force-free, i.e. $\left(\nabla \times \boldsymbol{B}_{\mathrm{e}}\right) \times \boldsymbol{B}_{\mathrm{e}}=$ 0 , so the equation of hydrostatic equilibrium of the external atmosphere is simply

$\nabla p_{\mathrm{e}}=\rho_{\mathrm{e}} \boldsymbol{g}$. 
(In the TM model, the external field is uniform, and hence also force free.) Using Eq. (3) and the equation of lateral pressure balance, (1), Eq. (2) can be rewritten as

$\rho \boldsymbol{v} \cdot \nabla \boldsymbol{v}=\left(\rho-\rho_{\mathrm{e}}\right) \boldsymbol{g}-\nabla\left(\frac{B_{\mathrm{e}}^{2}}{8 \pi}\right)+\frac{1}{4 \pi} \boldsymbol{B} \cdot \nabla \boldsymbol{B}$.

The term $\boldsymbol{v} \cdot \nabla \boldsymbol{v}$ in Eq. (4) can be decomposed into tangential and normal components as follows:

$$
\begin{aligned}
\boldsymbol{v} \cdot \nabla \boldsymbol{v}=v \frac{\mathrm{d}}{\mathrm{d} s}(v \sigma) & =\left(v \frac{\mathrm{d} v}{\mathrm{~d} s}\right) \sigma+v^{2} \frac{\mathrm{d} \sigma}{\mathrm{d} s} \\
& =\left[\frac{\mathrm{d}}{\mathrm{d} s}\left(\frac{v^{2}}{2}\right)\right] \sigma+v^{2} \frac{v}{R},
\end{aligned}
$$

where $\mathrm{d} \sigma / \mathrm{d} s=v / R$, where $R$ denotes the (signed) radius of curvature of the flux tube axis, given in terms of the height $h(x)$ or the angle $\theta(x)$ of the tube axis (see Fig. 1) by

$\frac{1}{R}=\frac{-\left(\mathrm{d}^{2} h / \mathrm{d} x^{2}\right)}{\left[1+(\mathrm{d} h / \mathrm{d} x)^{2}\right]^{3 / 2}}=-\cos \theta \frac{\mathrm{d} \theta}{\mathrm{d} x}$.

If we decompose the magnetic tension force $\boldsymbol{B} \cdot \nabla \boldsymbol{B} / 4 \pi$ in Eq. (4) into tangential and normal components in a similar way, we then find that the component of Eq. (4) in the normal ( $v)$ direction is

$\left(\frac{B^{2}}{4 \pi}-\rho v^{2}\right) \frac{1}{R}=-\left(\rho-\rho_{\mathrm{e}}\right) g \cos \theta+v \cdot \nabla\left(\frac{B_{\mathrm{e}}^{2}}{8 \pi}\right)$.

If the external magnetic field $\boldsymbol{B}_{\mathrm{e}}$ is uniform, as in the TM model, the term $v \cdot \nabla\left(B_{\mathrm{e}}^{2} / 8 \pi\right)$ in Eq. (7) vanishes and the normal component of the momentum equation reduces to

$\left(\frac{B^{2}}{4 \pi}-\rho v^{2}\right) \frac{\mathrm{d} \theta}{\mathrm{d} x}=\left(\rho-\rho_{\mathrm{e}}\right) g$.

In the case of the nonuniform force-free external field $\boldsymbol{B}_{\mathrm{e}}$ adopted by SJS, the term $v \cdot \nabla\left(B_{\mathrm{e}}^{2} / 8 \pi\right)$ in Eq. (7) does not vanish, but, as pointed out by SJS and Schlichenmaier (2003), its magnitude is small compared to the other terms in the equation, and hence Eq. (8) provides a good approximation.

Taking the scalar product of Eq. (2) and $\sigma$, and noting that the Lorentz force $(\nabla \times \boldsymbol{B}) \times \boldsymbol{B} / 4 \pi$ has no component along $\boldsymbol{\sigma}$, we find that the tangential component of the momentum equation is simply

$\rho \frac{\mathrm{d}}{\mathrm{d} s}\left(\frac{v^{2}}{2}\right)=-\frac{\mathrm{d} p}{\mathrm{~d} s}-\rho g \sin \theta=-\frac{\mathrm{d} p}{\mathrm{~d} s}-\rho g \frac{\mathrm{d} h}{\mathrm{~d} s}$.

Equations (8) and (9), the normal and tangential components of the steady momentum equation, provide the basis for our further discussion ${ }^{2}$.

\footnotetext{
2 The actual computed flows in the SJS and TM models also involve an energy equation describing heat transfer between the flux tube and its surroundings, but this equation is not necessary for our present discussion.
}

\section{The instability of the "serpentine" solutions of the SJS model}

The basic configuration of the serpentine solutions of Schlichenmaier $(2002,2003)$ is shown schematically in Fig. 2. Here we show that this configuration is unstable to displacements normal to the vertical plane in which the flux-tube axis lies. The consequence of this instability is that the serpentine configuration will not form in a vertical plane; instead, any meanderings will be primarily in a horizontal plane.

The terms on the left hand side of Eq. (8) represent the normal force on the flux tube due to the magnetic tension force and the centrifugal force, each of which is proportional to the curvature $1 / R$ of the tube axis. The term on the right-hand side represents the component of the buoyancy force normal to the flux tube, which can be directed either upward $\left(\rho<\rho_{\mathrm{e}}\right.$, positive buoyancy) or downward ( $\rho>\rho_{\mathrm{e}}$, negative buoyancy), depending on the values of $\rho$ and $\rho_{\mathrm{e}}$. In the serpentine flows of Schlichenmaier $(2002,2003)$, the flow speed becomes superAlfvénic (i.e., $v^{2}>B^{2} / 4 \pi \rho$ ) near the upstream footpoint and remains super-Alfvénic all along the serpentine configuration. Thus, the term in parentheses on the left-hand side of Eq. (8) is negative everywhere along the serpentine flux tube. It then follows from Eq. (8) that the flux tube is negatively buoyant $\left(\rho>\rho_{\mathrm{e}}\right)$ everywhere where the curvature of the tube axis is concave downward $(\mathrm{d} \theta / \mathrm{d} x<0)$ and positively buoyant $\left(\rho<\rho_{\mathrm{e}}\right)$ everywhere where the curvature is concave upward $(\mathrm{d} \theta / \mathrm{d} x>0)$. Thus, the elevated arches of the serpentine flux tube are heavier than their surroundings, and the descending (inverted) arches are lighter than their surroundings. (The negative buoyancy of an elevated arch in a serpentine flux tube is shown schematically in Fig. 4 of Schlichenmaier 2003.)

It is easy to see that such a configuration is unstable. Suppose the entire flux tube is tilted slightly while its entire axis remains in a single plane - i.e., imagine that the vertical plane containing the tube axis is rotated slightly about the midline, as shown in Fig. 2. In the tilted position, the magnetic tension and centrifugal forces lie in the tilted plane, but the buoyancy force remains vertical. Thus, the normal forces no longer balance, and indeed the buoyancy force now acts to increase the displacement. Both the negative buoyancy of the upper parts of the serpent and the positive buoyancy of the lower parts act to increase the tilt of the axial plane, and the whole serpentine configuration will tend to fall over on its side. Once the tube tilts over substantially (finite perturbation), the flow along the tube will begin to adjust, so we cannot follow the nonlinear development of the instability by simple arguments. However, it is clear from Eq. (8) that as long as the flow is super-Alfvénic, the buoyancy force won't be stably balanced.

The serpentine configurations of the SJS model were found, in spite of their inherent instability just demonstrated, because the two-dimensional computational scheme of the model restricts the tube axis to lie in a vertical plane. A fully threedimensional calculation would not lead to such a configuration. Short of doing such a calculation, we can imagine what might happen. Suppose we start with a static flux tube, as in the SJS calculations, and then drive the flow harder and harder by increasing the pressure at the upstream footpoint. The flux tube 
will remain in its "normal" configuration (see Fig. 1) until the flow speed becomes super-Alfvénic somewhere. At that point, the flux tube will indeed become unstable to kinking, because the centrifugal force will exceed the magnetic tension force locally, but the displacement will take place preferentially in the horizontal direction, thus avoiding having to do work against the opposing buoyancy forces. The kink instability will then lead to a serpentine equilibrium shape, but with the sinusoidal tube axis lying in a horizontal (rather than vertical) plane.

Finally, we point out that the arched siphon-flow flux tubes in the TM model are stable to the tilting of the axial plane. In these cases, with sub-Alfvénic flow, the curvature of the tube axis is everywhere concave downward, and the flux tube is positively buoyant everywhere. If the vertical plane containing the flux tube is tilted, the buoyancy provides a restoring force that will return the configuration to the vertical. Small lateral displacements of the flux tube axis in this case will produce buoyancy-driven oscillations about the equilibrium configuration, not an instability. The "normal" sub-Alfvénic configurations in the SJS model are also gravitationally stable.

\section{Conclusion}

One could conjecture that the very high, super-Alfvénic flow speeds required to produce the serpentine tube configurations of the SJS model are a consequence of the artificial outer boundary condition and would not occur in a real sunspot. However, even if such high flow velocities could be achieved, they will not lead to the vertical undulations found by Schlichenmaier $(2002,2003)$, because this configuration is unstable. Instead, any resulting undulations would form preferentially in a horizontal plane. As such, the serpentine model then no longer offers a possible explanation for Evershed downflows or outward-moving penumbral grains. The "normal" configuration in the SJS model also fails to explain the Evershed downflows, and the SJS model in general has all of the Evershed flow continuing outward along the magnetic canopy outside the sunspot, which seems to be contradicted by observations.

The arched siphon-flow model, exemplified by the TM model, does not suffer from the instability discussed here, and it offers a natural explanation of the cool, sometimes supersonic Evershed downflows. The arched configuration does require an additional mechanism to submerge and hold down the outer reaches of the flux tube. We have proposed that the required mechanism for the submergence is downward magnetic flux pumping by the turbulent granular convection just outside the sunspot (Thomas et al. 2002; Weiss et al. 2004). The TM model explains the salient features of the Evershed flow, which is concentrated in the darker penumbral filaments with their nearly horizontal magnetic fields, but it makes no attempt to also explain the moving penumbral grains, which instead may be associated with some sort of moving convective pattern in the brighter parts of the penumbra with their more vertical magnetic fields (Weiss 2002).

Acknowledgements. I thank Benjamin Montesinos, Rolf Schlichenmaier, and Nigel Weiss and for helpful comments.

\section{References}

Degenhardt, D. 1989, A\&A, 222, 297

Degenhardt, D. 1991, A\&A, 248, 637

Meyer, F., \& Schmidt, H. U. 1968, Z. Ang. Math. Mech., 48, T218

Montesinos, B., \& Thomas, J. H. 1997, Nature, 390, 485

Schlichenmaier, R. 2002, Astron. Nachr., 323, 303

Schlichenmaier, R. 2003, in Current Theoretical Models and High Resolution Solar Observations: Preparing for ATST, ed. A. A. Pevtsov, \& H. Uitenbroek, ASP Conf. Ser., 286, 211

Schlichenmaier, R., Jahn, K., \& Schmidt, H. U. 1998a, ApJ, 493, L121

Schlichenmaier, R., Jahn, K., \& Schmidt, H. U. 1998b, A\&A, 337, 897

Solanki, S. K. 2003, A\&AR, 11, 153

Spruit, H. C. 1981, A\&A, 98, 155

Thomas, J. H. 1988, ApJ, 333, 407

Thomas, J. H., \& Montesinos, B. 1990, ApJ, 359, 550

Thomas, J. H., \& Montesinos, B. 1991, ApJ, 375, 404

Thomas, J. H., \& Montesinos, B. 1993, ApJ, 407, 398

Thomas, J. H., \& Weiss, N. O. 2004, ARA\&A, 42, 517

Thomas, J. H., Weiss, N. O., Tobias, S. M., \& Brummell, N. H. 2002, Nature, 420, 390

Weiss, N. O. 2002, Astr. Nach., 323, 371

Weiss, N. O., Thomas, J. H., Brummell, N. H., \& Tobias, S. M. 2004, ApJ, 600, 1073 